\title{
Conjunctival impression cytology as an assessment of ocular surface changes following manual small incision cataract surgery
}

\author{
Pallamreddy S.L. ${ }^{1}$, Sandhya C.S. ${ }^{2}$, C. Jagannath ${ }^{3}$, K. Madhavi ${ }^{4}$ \\ ${ }^{1}$ Dr. Sree Lakshmi Pallamreddy, Associate Professor (D), ${ }^{2}$ Dr. C.S. Sandhya, Professor, HOD, ${ }^{3}$ Dr. C. Jagannath, \\ Associate Professor, ${ }^{4}$ Dr. K. Madhavi, Junior Resident; all authors are attached with Department of Ophthalmology, S V \\ Medical College, Tirupati (A.P.) India.
}

Corresponding Author: Dr. C.S. Sandhya, Professor \& HOD, Department of Ophthalmology, S.V. Medical College, Tirupati, Andhra Pradesh, India. E-mail: cheedella.sandhya@gmail.com

\begin{abstract}
Purpose: To determine the changes inocular surface following manual small incision cataract surgery. Setting: Department of Ophthalmology, Sri Venkateswara Medical College, Tirupati. Design: A prospective study Methods: The study was conducted in 100 eyes of 100 patients. All selected patients without dry eye symptoms preoperatively and who underwent uncomplicated cataract surgery were included in the study. They were studied for tear film breakup time (TBUT), ocular surface staining by Rose Bengal (RB) staining method, and conjunctival impression cytology (CIC) from inferior bulbar conjunctiva. Tests were performed 1 day before and $1 \mathrm{wk}$, 1 month and 3 months after manual small incision cataract surgery (SICS). Results: Majority of the study participants were aged between 51- 60 years (51\%) with the female predominance. In $8 \%$ of the patients, lower TBUT values were recorded at 3 months postoperative follow up, with statistical significance $(\mathrm{P}<0.01)$. There was a statistically significant difference in ocular surface changes with $\mathrm{RB}$ staining between preoperative and postoperative 1 week, 1 month, and 3 months follow up $(\mathrm{P}<0.002)$. On $\mathrm{CIC}, 12 \%$, $49 \%, 46 \%$ of patients showed abnormal grades (grade 2 or 3 ) at 1 week, 1 month, and 3 months postop respectively with a statistically significant value $(\mathrm{P}<0.03)$. Conclusion: Patients undergoing manual SICS without having dry eye preoperatively, showed changes in ocular surface and tear film stability. Positive test with RB staining and abnormal grading of goblet cells studied by CIC during the postoperative period had shown that compromised ocular surface health after SICS, which can produce dry eye.
\end{abstract}

Keywords: CIC, TBUT, SICS, Ocular surface, Impression cytology

\section{Introduction}

Dry eye is a common condition affecting the tear film and ocular surface. The 2007 International Dry Eye Workshop stated that "dry eye is a multifactorial disease of the tear film and ocular surfacethat results in symptoms such as ocular discomfort, visual disturbance, and potential damage to the ocular surface". [1] Conjunctival Goblet cells, the chief source of mucinis most dense nasally, least dense in the upper temporal fornix and absent at the palpebral mucocutaneous junction and limbus. "The density of goblet cells is $10 \pm 3$ cells $/ \mathrm{mm}^{2}$ [2]. In developing countries like India, manual SICS with intraocular lens implantation is considered as a safe surgical procedure which provides good postoperative outcomes.However, after cataract

Manuscript received: $28^{\text {th }}$ July 2019

Reviewed: $8^{\text {th }}$ August 2019

Author Corrected: $17^{\text {th }}$ August 2019

Accepted for Publication: $23^{\text {rd }}$ August 2019 surgery, many patients complain of irritation, foreign body sensation, redness, blurring of vision which is considered as unwanted effects of the surgery [3].

Although the precise mechanism(s) for postoperative dry eye is not completely understood, past studies have suggested that eyes with this type of abnormality have a short TBUT [4], a decrease in the density of goblet cells [5], as seen in eyes with conventional dry eye syndrome [6], A decrease in goblet cell density will also reduce mucin secretion and disrupt the tear film stability consequently lead to dry eye.

Purpose of this study was to determine the effects of small incision cataract surgery on the ocular surface assessed by conjunctival goblet cells with impression cytology, causing the tear-film instability and dry eye. 
Original Research Article

\section{Materials and methodology:}

Study setting- The study was conducted in the Department of Ophthalmology, Sri Venkateswara Medical College, Tirupati.

Study Design- A prospective study

Study period- The study period was between December 2014 and June 2016. Ethical committee approval was obtained from the institutional ethical committee (IEC). Written informed consent was taken from all patients included in the study.

Inclusion criteria: All patients who were above 50 years of age and who underwent uneventful manual SICS were included in the study.

Exclusion criteria: The patients with pre-existing dry eyes, rheumatoid arthritis, Sjogren's syndrome and other autoimmune disorders and patients with pre-existing ocular diseases like uveitis, glaucoma, ocular allergies, disorders of the lid or the nasolacrimal pathway, pterygium and previous ocular surgeries and patients with surgical complications were excluded from the study.

Clinical examinations included comprehensive and extensive anterior segment evaluation done under slit lampto excludeany pre-existing ocular surface disease. TBUT, RB staining, and CIC were done. The patients were started on topical and systemic antibiotics one day before surgery. On the day of surgery, $0.8 \mathrm{mg}$ tropicamide, and $10 \%$ phenylephrine eye drops were used to dilate pupils 90 minutes before cataract surgery.

Manual SICS was performed under 2 point peribulbar block. The standard incision was 6 to $6.5 \mathrm{~mm}$ in length and 1.5 to $2 \mathrm{~mm}$ from the limbus. Implantation of a rigid PMMA intraocular lens was done in all the patients. A standard postoperative regime was followed for all the patients. It included the use of steroid-antibiotic combination in tapering doses for eight weeks. Postoperative evaluation was done at 1week, 1month, and 3 months. At each visit TBUT, RB staining, CIC was done.

Tear film break up time measurement (TBUT): The TBUT measures the interval between the last complete blink and the first appearance of the dry spot over cornea using cobalt blue filter after application of $2 \%$ fluorescein dye under slitlamp biomicroscopy. Three TBUT readings were noted, and an average of the readings was calculated.If TBUT less than 10 seconds it is considered as adry eye.

Rose Bengal stain (RB): It is a measure of assessing ocular surface change using the Rose Bengal dye.A commercially available, sterile Rose Bengal strip moistened with 4\% Xylocaine solution was applied to the inferior cul de sac.The eye was examined after15 sec for staining of conjunctiva and cornea under a bright light or red-free light under the slit lamp. Van Bijsterveld scoring system was used to grade, based on a scale of 0-3 in 3 areas: cornea, nasal conjunctiva, and temporal conjunctiva. A quantitative scale of 0 to 3 was used in each area of the eye. An additive score of 4 or more in the eye considered a positive test for dryness of ocular surface.

Conjunctival impression cytology (CIC): Conjunctival impressions were taken from inferior bulbar conjunctiva. Procedure: The test was conducted using cellulose acetate strips having a pore diameter of $0.45 \mu \mathrm{m}$ with the patient in supine position.A drop of $4 \%$ lignocaine was instilled in the eye. After inserting a wire speculum, Millipore cellulose acetate filter strips were cut into $3 \times 10 \mathrm{~mm}$ size pieces with a diagonal edge and were applied on the inferior-nasal bulbar conjunctiva with the help of a blunt smooth-edged forceps. The specimens were collected from the inferior bulbar conjunctiva. The rough surface of the paper was used, and it was pressed gently by a smooth glass rod. The strip was removed with a peeling motion after 5 seconds. The papers were fixed in a fixative solution (absolute alcohol + glacial acetic acid $+40 \%$ formaldehyde in 20:1:1 respectively) and stained with PAS (periodic acid Schiffers) and counterstained with hematoxylin. The filter paper was mounted and examined under the microscope and graded. Nelson grading system [7] was followed for staging. Interpretation: The eyes having grade 2 or 3 changes were considered positive for dry eye. Eyes with grade 0 or grade 1 change were considered negative [Figure 1, 2, 3]. 


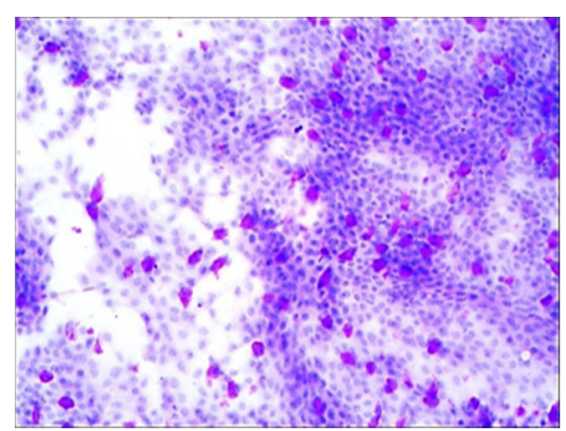

Figure-1: Microscopic picture showing grade 1 goblet cells

\section{Original Research Article}

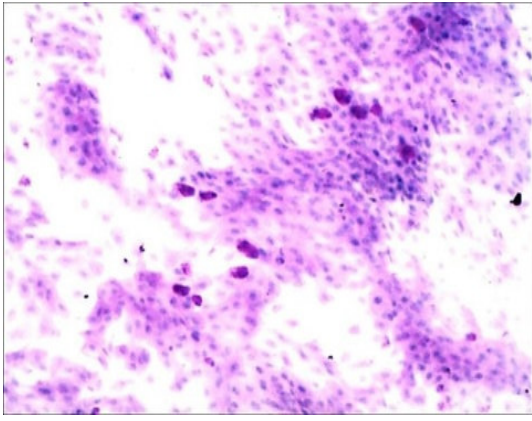

Figure-2: Microscopic picture showing grade 2 goblet cells

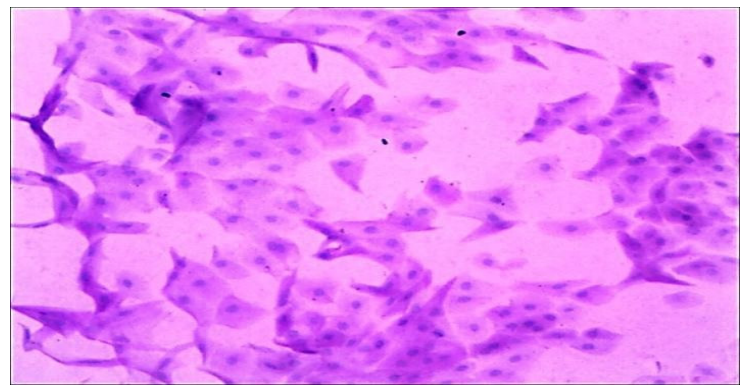

Figure-3: Microscopic picture showing Grade 3: Goblet cells

Statistical analysis: Chi-square test was applied to find significance among qualitative variables. Paired t-test was applied to find significance among quantitative variables and to check preoperative and postoperative results. Descriptive statistics were applied to quantitative variables, Mean and S.D. The Statistical software SPSS 20.0 was used for analyzing the data and Microsoft Word, and Excel 2011 has been used to generate graphs and tables.

\section{Results}

All the patients were followed for 3 months postoperatively and assessed for the tear film stability by TBUT, RB staining of the ocular surface and conjunctival goblet cells based on impression cytology. The results of the study were analyzed as below.

In the present study majority (51\%) of the study, participants were aged between 51 to 60 years [Figure-4]. Females $(59 \%)$ outnumbered males $(41 \%)$.

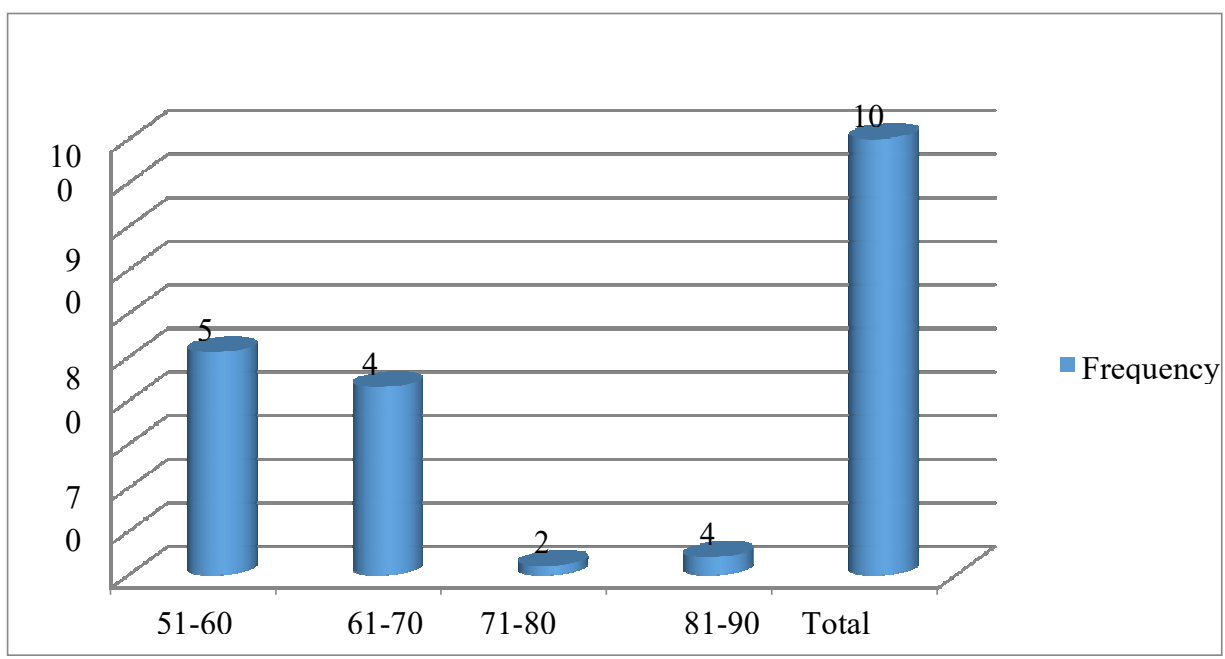

Fig-4: Distribution of study subjects according to age. 
The TBUT analysis showed that the preoperative TBUT mean $\mathbf{2 0 . 7 2} \pm \mathbf{5 . 3 7 1}$ and at postoperative one week, one month and 3 months $6 \%, 23 \%$, and $8 \%$ had low TBUT values respectively. Changes in TBUT were found to be statistically significant through all postoperative periods. In the present study, no patient in the preoperative group had TBUT values in the dry eye range, whereas $6 \%, 23 \%$ and $8 \%$ of patients in the postoperative period ( 1 week, 1 month, and 3 months respectively) had TBUT values in the dry eye range. $(\mathrm{p}<0.01)$ [Table-1].

Table-1: Comparison of TBUT at Pre-op, Post-op at 1 week, 1 month and 3 months (F: Frequency)

\begin{tabular}{|c|c|c|c|c|c|c|c|c|c|c|}
\hline \multirow[t]{2}{*}{ TBUT } & \multicolumn{2}{|c|}{ Pre-op } & \multicolumn{2}{|c|}{ Post-op at 1 week } & \multicolumn{2}{|c|}{ Post-op at 1 month } & \multicolumn{2}{|c|}{$\begin{array}{c}\text { Post-op at } 3 \\
\text { months }\end{array}$} & \multirow{2}{*}{$\begin{array}{c}\text { t- } \\
\text { value }\end{array}$} & \multirow[t]{2}{*}{ p-value } \\
\hline & $\mathbf{F}$ & $\operatorname{Mean} \pm$ SD & $\mathbf{F}$ & $\operatorname{Mean} \pm$ SD & $\mathbf{F}$ & Mean \pm SD & F & Mean \pm SD & & \\
\hline $\begin{array}{l}>=10 \\
\text { Normal }\end{array}$ & 100 & $20.72 \pm 5.371$ & 94 & $17.71 \pm 5.028$ & 77 & $15.15 \pm 4.21$ & 92 & $17.71 \pm 4.86$ & \multirow{3}{*}{8.33} & \multirow{3}{*}{$\mathbf{P}<\mathbf{0 . 0 0}$} \\
\hline $\begin{array}{l}<10 \\
\text { Dry }\end{array}$ & 0 & 0 & 6 & $8 \pm 1.09$ & 23 & $7.30 \pm 1.49$ & 8 & $8 \pm 1.85$ & & \\
\hline Total & 100 & & 100 & & 100 & & 100 & & & \\
\hline
\end{tabular}

Ocular surface staining with Rose Bengal stain showed that before surgery, all patients had a rose Bengal stain grading of 0 , none of them had abnormal grading. Post-surgerygrading increased to 3 months follow up with statistically significant value at each period [Figure-5].

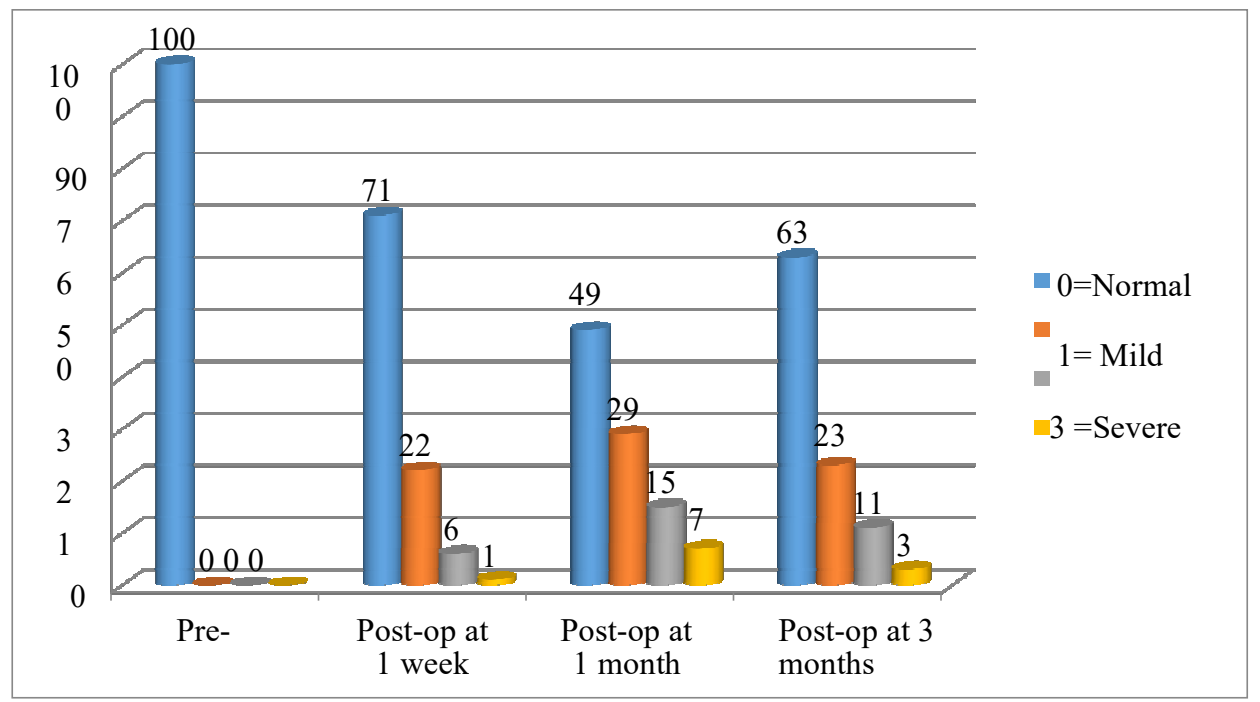

Figure-5: Multiple bar diagram showing RB grades at Pre-op, Post- op at 1 week, 1 month and 3 months

CIC analysis based on Nelson grading system, showed normal grading (grade 0 or 1) preoperatively in all patients, whereas $12 \%, 49 \%, 46 \%$ of patients showed abnormal grades (grade2 or 3 ) at post op 1 wk, 1 month, and 3 months respectively with a statistically significant value $(\mathrm{P}<0.03)$. [Table -2$]$

Table-2: Comparison of CIC at Pre-op, Post-op at 1 week,1 month and 3 months.

\begin{tabular}{|c|c|c|c|c|c|}
\hline CIC & Pre-op & $\begin{array}{c}\text { Post-op at } \mathbf{1} \\
\text { week }\end{array}$ & $\begin{array}{c}\text { Post-op at } 1 \\
\text { month }\end{array}$ & $\begin{array}{c}\text { Post-op at } \\
\text { 3 months }\end{array}$ & P - value \\
\hline $0,1=$ Normal & 100 & 88 & 51 & 54 & \multirow{2}{*}{$\mathrm{P}<0.03$} \\
\hline $1,2=$ Abnormal & 0 & 12 & 49 & 46 & $\mathbf{1 0 0}$ \\
\hline Total & $\mathbf{1 0 0}$ & $\mathbf{1 0 0}$ & $\mathbf{1 0 0}$ & \\
\hline
\end{tabular}




\section{Discussion}

A stable and smooth tearfilm is essential for the optimal function of the cornea as a refractive unit of the eye. Dry eye syndrme is a multifactorial disease of the tear film, and ocular surface, which results in visual disturbance, symptoms of discomfort, tear instability with potential damage to the ocular surface. Cataract surgery is known to disrupt the ocular surface and alter the tearfilm quantitatively and qualitatively $[3,4]$.

The present studyshowed that manual SICS influences the ocular surface changes and thereby tearsfilm instability. The prospective study was conducted on 100 patients who underwent SICS. For these 100 patients, various parameters were used to assess the tear film stability preoperatively and postoperatively at 1 week, 1 month, and 3 months. Out of the 100 patients who underwent SICS, 95\% belonged to 51-70 years age group. Females (59\%) were more compared to males $(41 \%)$.

Concerning the tear film break up time (TBUT). In the present study, the TBUT in all the patients preoperatively was in the normal range. During the post-op period, $6 \%$ of the patients, $23 \%$ of the patients and $8 \%$ of the patients at $1 \mathrm{wk}, 1 \mathrm{month}$ and 3 monthsrespectively had TBUT valuesin dry eye range $(p<0.01)$. The lowest value of TBUT was noticed at 1 month, and after 3 months there was a slight improvement in value again.

Chandan $\mathrm{C}$ et al [8], and Mohana $\mathrm{S}$ et al [9], in their study also found a lower value of TBUT at 1 month follow up. In a study by Chandan $\mathrm{C}$ et al. [8], TBUT values decreased in the postoperative period to statistically significant levels. In a study by Mohana S et al [9], TBUT values significantly decreased at 1 week, 1 month, and reached near normal values at 3 months. In the present study, $92 \%$ of the patients showed normal TBUT values at 3 months postoperative period. In a study by Li XM et al [4], Srinivasan R et al [10] Liu Z et al [11], and Ram et al [12], it had shown that significant decreasein TBUT value post operatively after phacoemulsification. In a study by Neda A et al [13], which was done to examine the dry eye after cataract surgery, there was a decrease in TBUT values postoperatively.

Ocular surface staining in the present study by the Rose Bengal staining did not show any abnormality in any of the patients, where as $29 \%, 51 \%$ and $37 \%$ of patients in the postoperative 1 week, 1 month, and 3 months respectively had abnormal $\mathrm{RB}$ staining. In a study by Oh et al [5], RB staining showed a similar pattern. In a study by Yusuf Rizvi et al [14], Corneal staining studied by fluorescein stain scores differed significantly frombaseline values in the postoperativeperiod of one month. Corneal staining pattern differed in the SICS group but notthe phacoemulsification group at day 60 .

However Staining scores were not significantly different from pre-operative levels at day 90, for SICS aswell as phacoemulsification group suggesting aquiescent surface. This is similar to the studies conducted by Li, et al [4] and Ram, et al [12]. However, Liu et al [11], found that fluorescein staining increased in the 1st-week follow-up visits and decreased in the next visits subsequently. In a study by P.K. Sahu et al [15], showed that postoperatively, the lissamine grading increased till 1-month, then found a reversal trend, with statisticallysignificant values at each period.

In the present study, the CIC assessmentshowed significant changes postoperatively. CIC showed abnormality in none of the cases preoperatively, whereas $12 \%, 49 \%$ and $46 \%$ of cases showed abnormality at postoperative 1 week, 1month, and 3 months, respectively $(\mathrm{p}<0.03)$. Similar to the present study, Oh T et al [5], had shown that mean goblet cell density reduced significantly at 1 day, 1 month and 3 months postoperatively $(\mathrm{p}<0.01)$.

In a study by Li et al [4], CIC demonstrated that even 3 months after the cataract surgery, goblet cells were reduced along with squamous metaplasia which suggests that dry eye might have been induced by eye drops. In a study by Mohan $\mathrm{S}$ et al, mean number of goblet cells found in CIC was 214.92, and the standard deviation was 54.59 preoperatively in the control group. At the end of 3 months mean a number of goblet cells did not show much change in the control group whereas in the study group number of goblet cells decreased significantly from $223.37 \pm 148.61$ to $163.79 \pm 61.47$ at 3 months [9].

The changes in ocular surface and tear film function following cataract surgery causing dry eye to the patient may be due to mechanisms like chronic use of eye drops following the cataract surgery due to the presence of preservatives [16], tear film irregularity at the site of incision which causes tear film to break up faster or the mucin production from conjunctiva may be decreased, exposure to light from operating microscope [3, 17], misuse of eye drops [18, 19], decreased corneal 


\section{Original Research Article}

sensations (a study by Donnenfeld ED et al [20], disrupting the corneal lacrimal gland loops resulting in decreased tear secretion and the incision might result in severing of the corneal nerves which cause decreased corneal sensation (a study by Kohlhaas M et al [21]

The major limitations of the present study were shortterm follow up (3 months), long-term effects were not evaluated., The subjective symptoms of dry eye OSDI scoring were not considered as part of the study. The effect of preservative free eye dropsafter cataract surgery on the ocular surface was not studied.

\section{Conclusion}

The present study suggests that manual SICS with Corneo-scleral incision has effects on the ocular surface and tear film stability. Goblet cell study by impression cytology showed abnormal grading postoperatively compared topre-op grading. The decrease in goblet cell density and altered morphology had not recovered at 3 months post op of cataract surgery. Therefore, ocular surface damage during and after cataract surgery appears to be one of the pathological factor causing ocular discomfort and dry eye syndrome after cataract surgery with corneo-scleral incision.

Ocular surface instability is the leading cause of dissatisfaction among cataract patients postoperatively. Dry eye is the disease of the ocular surface and the tear film, which results in symptoms such as ocular discomfort, visual disturbanceandpotential damage to the ocular surface.

Because cataract surgery has been shown to exacerbate orinduce pre-existing ocular surface changes and dry eye, it is of significanceto physicians to be vigilant of the syndrome while evaluating cataract patients, to plan surgery accordingly and treat the condition preoperatively and post operatively.

The improved stability and quality of the tearfilm results in a regular ocular surface and improve refractive outcomes after cataract.

\section{What the study adds to the existing knowledge?}

The present study, Conjunctival impression cytology as an assessment of ocular surface changes following manual small incision cataract surgery suggests that CIC is more sensitive for evaluation of ocular surface changes and detect early subtle changes in the ocular surface in post-cataract surgery patients.

\section{Author's contribution}

Dr. SreeLakshmi Pallamreddy: Design of the study, Data collection, Data analysis and interpretation, Drafting the article, Revision of the article, Approval of the manuscript to be published.

Dr. C.S. Sandhya: Design of the study, Data analysis and interpretation, Drafting the article, Revision of the article, Approval of the manuscript to be published

Dr. C. Jagannath: Data analysis and interpretation, Revision of the article, Approval of the manuscript to be published

Dr. K. Madhavi: Design of the study, Data collection, Data analysis and interpretation, Approval of the manuscript to be published

Acknowledgments: Nil

Funding: Nil, Conflict of interest: Nil

Permission from IRB: Yes

\section{References}

1. The definition and classification of dry eye disease: report of the Definition and Classification Subcommittee of the International Dry Eye WorkShop (2007). Ocul Surf. 2007;5(2):75-92. doi: https://doi.org/ 10.1016/S1542-0124(12)70081-2

2. Cibis GW. Fundamentals and principles of ophthalmology. Basic \& Clinical Science Course. American Academy of Ophthalmology. Section 2; LEO Publishers; 2004-2005 :309-317.

3. Cho YK, Kim MS. Dry eye after cataract surgery and associated intraoperative risk factors. Korean J Ophthalmol. 2009; 23(2):65-73. doi:10.3341/kjo. 2009. 23. 2.65 .

4. Li XM, Hu L, Hu J, Wang W. Investigation of dry eye disease and analysis of the pathogenic factors in patients after cataract surgery. Cornea. 2007;26 (9 Suppl 1):S16-S20. doi:10.1097/ICO.0b013e31812f67ca

5. Oh T, Jung Y, Chang D, Kim J, Kim H. Changes in the tear film and ocular surface after cataract surgery. Jpn J Ophthalmol. 2012;56(2):113-8 . doi: 10.1007/ s10384- 012-0117-8.

6.Murube J, Rivas L. Impression cytology on conjunctiva and cornea in dry eye patients establishes a correlation between squamous metaplasia and dry eye clinical severity. Eur J Ophthalmol. 2003; 13(2): $115-127$ 


\section{Original Research Article}

7. Nelson JD, Wright JC. Conjunctival goblet cell densities in ocular surface disease. Arch Ophthalmol. 1984;102 (7): 1049-1051.doi: 10.1001/archopht.1984. 01040030851031

8. Chakraborti C, Mukhopadhyay U, Mondal M, Sinha M. Dry eye disease following manual small incision cataract surgery: A study from eastern India. Indian J App Res, 2016;6(8):270-273. doi: 10.15373/2249555x

9. Sinha M, Sinha A, Chowdhury B. Comparative evaluation of dry eye following cataract surgery: a study from north india.IOSR J Dent MedSci.2014;13(6):13-18

10. Srinivasan R, Agarwal V, Suchismitha T, Kavitha. Dry eye after phacoemulsification.AIOC,2008:116-118.

11. Liu Z, Luo L, Zhang Z, Cheng B, Zheng D, Chen $\mathrm{W}$, et al. Tear film changes after phacoemulsification. Zhonghua Yan Ke Za Zhi. 2002;38(5):274-277.

12.Ram J, Gupta A, Brar G, Kaushik S, Gupta A. Outcomes of phacoemulsification inpatients with dry eye. J Cataract Refract Surg. 2002;28(8):1386-1389. doi: https://doi.org/10.1016/S0886-3350(02)01387-1

13. Afsharkhamseh $\mathrm{N}$, Movahedan A, Motahari $\mathrm{H}$, Djalilian AR. Cataract surgery in patients with ocular surface disease: an update in clinical diagnosis and treatment. Saudi J Ophthalmol. 2014;28(3):164-167. doi:10.1016/j.sjopt.2014.06.013.

14. Rizvi Y, Singh S, Dokania A. Comparative assessment of tear function and ocular surface following cataract surgery employing manual SICS and phacoemulsification techniques. Indian $\mathrm{J}$ Basic App Med Res. 2014;4(1):544-553.
15. Sahu PK, Das GK, Malik A, Biakthangi L. Dry eye following phacoemulsification surgery and its relation to associated intraoperative risk factors. Middle East Afr J Ophthalmol. 2015; 22(4):472-477. doi: 10.4103/ 0974- 9233.151871

16. Walker TD. Benzalkonium toxicity. Clin Exp Ophthalmol. 2004;32(6):657.DOI:10.1111/j.1442-9071. 2004. 00922.x

17. Khanal S, Tomlinson A, Esakowitz L, Bhatt P, Jones D, Nabili S, et al. Changes in corneal sensitivity and tear physiology after phacoemul-sification. Ophthalmic Physiol Opt. 2008; 28(2):127-134. doi: 10.1111/j.1475-1313.2008.00539.x.

18. Lyne A. Corneal sensitivity after surgery. Trans Ophthalmol Soc UK. 1982;102 (pt 2):302-305.

19. Zabel RW, Mintsioulis G, MacDonald IM, Valberg J, Tuft SJ. Corneal toxic changes after cataract extraction. Can J Ophthalmol. 1989;24(7):311-316.

20. Donnenfeld ED, Solomon K, Perry HD, Doshi SJ, Ehrenhaus M, Solomon R, et al. The effect of hinge position on corneal sensation and dry eye after LASIK. Ophthalmol. 2003;110(5): 1023-1029. doi:10.1016/ S0161 -6420(03)00100-3

21. Kohlhaas M. Corneal sensation after cataract and refractive surgery. J Cataract Refract Surg. 1998;24 (10): 1399-1409. doi: https://doi.org/10.1016/S0886$3350(98) 80237-\mathrm{X}$

\section{How to cite this article?}

Pallamreddy S.L, Sandhya C.S, C. Jagannath, K. Madhavi. Conjunctival impression cytology as an assessment of ocular surface changes following manual small incision cataract surgery. Trop J Ophthalmol Otolaryngol.2019;4 (4): 263-269.doi:10.17511/jooo.2019.i04.02 